\title{
OPEN Multimodal integrated approaches in low grade glioma surgery
}

\author{
Tamara lus ${ }^{1 凶}$, Edoardo Mazzucchi ${ }^{2,3}$, Barbara Tomasino ${ }^{4}$, Giada Pauletto ${ }^{5}$, \\ Giovanni Sabatino 2,3 , Giuseppe Maria Della Pepa ${ }^{2}$, Giuseppe La Rocca ${ }^{2,3}$, Claudio Battistella ${ }^{6}$, \\ Alessandro Olivi ${ }^{2} \&$ Miran Skrap ${ }^{1}$
}

Surgical management of Diffuse Low-Grade Gliomas (DLGGs) has radically changed in the last 20 years. Awake surgery (AS) in combination with Direct Electrical Stimulation (DES) and real-time neuropsychological testing (RTNT) permits continuous intraoperative feedback, thus allowing to increase the extent of resection (EOR). The aim of this study was to evaluate the impact of the technological advancements and integration of multidisciplinary techniques on EOR. Two hundred and eighty-eight patients affected by DLGG were enrolled. Cases were stratified according to the surgical protocol that changed over time: 1 . DES; 2 . DES plus functional MRI/DTI images fused on a NeuroNavigation system; 3. Protocol 2 plus RTNT. Patients belonging to Protocol 1 had a median EOR of $83 \%$ (28-100), while those belonging to Protocol 2 and 3 had a median EOR of $88 \%$ (34-100) and $98 \%(50-100)$ respectively $(p=0.0001)$. New transient deficits with Protocol 1,2 and 3 were noted in $38.96 \%, 34.31 \%$ and $31,08 \%$ of cases, and permanent deficits in $6.49 \%, 3.65 \%$ and $2.7 \%$ respectively. The average follow-up period was 6.8 years. OS was influenced by molecular class $(p=0.028)$, EOR $(p=0.018)$ and preoperative tumor growing pattern $(p=0.004)$. Multimodal surgical approach can provide a safer and wider removal of DLGG with potential subsequent benefits on OS. Further studies are necessary to corroborate our findings.

Adult Diffuse Low-Grade Gliomas (DLGG) are rare genetically heterogeneous tumors accounting for up to 7\% of primary brain tumors. DLGG generally affect young adults with no or only slight functional disorders.

Patients with DLGG show an initial subclinical phase, followed by continuous tumor growth, infiltration of eloquent areas, progression to a higher grade of malignancy, which leads to neurological disability and death.

The management of LGGs has radically changed over the time: from a "wait and see" attitude to maximal safe resection as the first step in DLGGs workflow ${ }^{1-12}$. To achieve this purpose, a personalized anatomo-functional planning and intraoperative strategy are crucial to have an optimal balance between a maximal and safe resection.

Because functional neuroimaging tends to show limited reliability, intraoperative direct electrical stimulation (DES), especially in awake patients benefiting from real-time cognitive monitoring, is the best way to increase the extent of resection while sparing eloquent neural networks ${ }^{13}, 14$.

Awake surgery (AS) represents the gold standard for cerebral brain mapping considering that, to date, this is the only technique that permits a direct identification of neural networks ${ }^{15}, 16$. Recent studies have shown that awake mapping in association with real-time neuropsychological testing (RTNT), which reduces the uncertainty of negative mapping, resulted in higher extent of resection (EOR) and preservation of quality of life both for DLGGs involving language and extra-language functional networks ${ }^{15-17}$. Moreover, modern preoperative and intraoperative imaging techniques, along with surgical tools and developments in monitoring techniques, have advanced the potential magnitude of resection in eloquent areas ${ }^{18}$.

These previous studies addressed the impact of each single approach on clinical and EOR parameters ${ }^{15-17}$, but to the best of our knowledge, there are no studies based on the combinatory effect of different techniques.

The principal aim of this investigation was to analyze the effect on the EOR, and consequently on OS, of the evolution of intraoperative techniques, also considering the recent advances of the knowledge of molecular features of DLGG.

\footnotetext{
${ }^{1}$ Neurosurgery Unit, Department of Neurosciences, Santa Maria della Misericordia University Hospital, Piazzale Santa Maria della Misericordia, 15, 33100 Udine, Italy. ${ }^{2}$ Institute of Neurosurgery, Fondazione Policlinico Gemelli, Catholic University, Rome, Italy. ${ }^{3}$ Department of Neurosurgery, Mater Olbia Hospital, Olbia, Italy. ${ }^{4}$ RCCS "E. Medea," Polo Regionale del FVG, San Vito al Tagliamento, Pordenone, Italy. ${ }^{5}$ Neurology Unit, Department of Neurosciences, Santa Maria della Misericordia University Hospital, Udine, Italy. ${ }^{6}$ Department of Medicine, University of Udine, Udine, Italy. ${ }^{\varpi}$ email: tamara.ius@gmail.com
} 


\section{Materials and methods}

Study population. Data from adult patients who underwent surgery for DLGG in a single institution between 2000 and 2018 were retrospectively analyzed.

Patients were enrolled according to the following criteria:

- Age $\geq 18$ years

- Preoperative MRI suggestive of supratentorial LGG near or involving the cortical and subcortical eloquent areas

- No previous surgery

- No preoperative chemo- or radiotherapy

- At least 18 months of follow-up

- Objective evaluation of EOR on MRI images in DICOM format based on T2-weighted MRI sequences

- Revision of histopathological specimens according to the 2016 WHO Classification of Tumors of the Central Nervous System

- Intraoperative brain mapping and neurophysiological monitoring.

Needle biopsies were excluded from the study.

The tumor involvement of a cortical or subcortical eloquent was detected according to the relationships of DLGG to eloquent regions of the brain as predicted by the preoperative magnetic resonance imaging scan, functional MRI and Diffusion Tensor Imaging (DTI) data.

The local Ethics Committee, Comitato Etico Unico Regionale del Friuli Venezia Giulia, approved this investigation (protocol N.0036567/P/GEN/EGAS, ID study 2540). Considering that the study was retrospective, written consent to participate in the study was not applicable. Written informed consent was obtained for surgery. Consent in this form was decided and approved by the local ethics committee Regione Friuli Venezia Giulia, Italy. All research was performed in accordance with the Declaration of Helsinki.

Intraoperative surgical protocol. The surgical procedures were conducted under cortical and subcortical white matter brain mapping, according to the intraoperative technique previously described ${ }^{19}$.

The AS protocol was selected following the standard protocol in use during that specific time period at our institution ${ }^{17}$. In addition to DES, RTNT was applied in the last series during $\mathrm{AS}^{17}$.

In this investigation, the data were collected over about 20 years (from 2000 to 2018), during which the intraoperative technical protocol changed.

The following 3 consecutive intraoperative protocols were used during this time period:

- Protocol 1 (January 2000-December 2004): patients underwent surgery with the aid of cortico-subcortical DES, neurophysiological monitoring (motor evoked and somatosensorial potential), and intraoperative use of the NeuroNavigation (NN) system.

- Protocol 2 (January 2005-to present): Protocol 1 plus fMRI/DTI data on the T1-weighted/T2-weighted 3D MRI studies for intraoperative NN.

- Protocol 3 (January 2011-to present): Protocol 2 plus RTNT in AS.

Patients belonging to Protocol 1 and 2 were operated on AS or General Anesthesia (GA) based on the specific clinical and neuropsychological status of each subject.

Volumetric analysis. Magnetic resonance images in DICOM format were used to assess both pre- and postoperative tumor volume by using axial preoperative post-contrast T1-weighted MRI slices, pre and postoperative axial T2-weighted MRI studies. All pre- and postoperative tumor segmentations were performed manually across all MRI slices using the OsiriX software tool ${ }^{20}$.

The preoperative tumor growing pattern (infiltrative vs. expansive) was expressed as $\Delta \mathrm{T} 2 \mathrm{~T} 1$ index that is preoperative volumetric tumor volume on T2-weighted MRI images-preoperative volumetric tumor volume on T1-weighted images ${ }^{6,20}$. As previously described, higher levels of preoperative $\Delta T 2 T 1$ MRI index represent the prevalence of the diffusive growing mechanism with the tendency to infiltrate the functional cortical areas and subcortical pathways, thus limiting the achievable resection.

The postoperative EOR was evaluated by using 3D T2-weighted MRI axial images as follows: (pre-operative tumor volume - post-operative tumor volume)/(pre-operative tumor volume $)^{20}$.

Histological and molecular analysis. All histological samples were reviewed according to the 2016 World Health Organization (WHO) classification ${ }^{21}$. Molecular markers were evaluated as previously described ${ }^{22}$.

Statistical analysis. Statistically significant differences on distribution were evaluated performing chisquared test for categorical variables, and t-test, Wilcoxon rank-sum tests or Kruskal-Wallis for continuous variables, as appropriate.

Overall Survival (OS), progression free survival (PFS) and malignant progression free survival (MPFS) were estimated using the Kaplan-Meier approach. Univariate and multivariate Cox regression models were performed to identify the association between any variable and OS, PFS and MPFS as outcome variables, after the proportional hazards assumption had been verified.

The primary endpoints were EOR and OS differences among the three groups. 
Retention in the stepwise model required the variable to be significant to a level of $p=0.05$ in a multivariate analysis ${ }^{23}$.

The Spearman's rank correlation coefficient was computed to define the relationship between pre-operative $\triangle \mathrm{T} 2 \mathrm{~T} 1 \mathrm{MRI}$ index and postoperative residual tumor computed on T2-weighted MR images.

All analyses were conducted using Stata/SE software (version 14.0 Stata Corp.), and data were presented as HRs and $95 \%$ CIs.

Ethical approval. The local Ethics Committee, Comitato Etico Unico Regionale del Friuli Venezia Giulia, approved this investigation (protocol N.0036567/P/GEN/EGAS, ID study 2540).

Informed consent. Considering that the study was retrospective, written consent to participate in the study was not applicable. Written informed consent was obtained for surgery.

\section{Results}

Study population. Three hundred and fifty-two adult patients underwent surgery at our institution for primary supra-tentorial LGGs. A total of 288 patients met the inclusion criteria. Patient demographic, clinical and radiological data are summarized in Table 1. According to clinical and neuropsychological status of each subject, AS was selected in 175 cases, GA was used in 113 cases.

Patients were surgically treated as follows:

- Protocol 1: 77 patients (26.74\%).

- Protocol 2: 137 patients (47.57\%).

- Protocol 3: 74 patients (25.69\%).

Overall, new postoperative deficits were noted in a total of 100 patients (34.72\%). The incidence of permanent postoperative deficits was relatively low in all protocol groups. Post-surgical deficits were stratified according to each surgical protocol (Table 2).

In detail, new deficits with Protocol 1, 2 and 3 were noted respectively in $38.96 \%, 34.31 \%$ and $31.08 \%$ of cases in the immediate post-operative period and in $6.49 \%, 3.65 \%$ and $2.7 \%$ of patients 6 months after surgery.

No intra or postoperative mortality was observed.

EOR and surgical protocol. The median EOR was $90 \%(28-100)$ in the study population. The mean EOR resulted to be statistically different between patient operated with AS when compared to those operated under GA $(p=0.006)$. Among patients operated on AS, mean EOR was higher in those in which the Protocol 3 was applied ( $p=0.001$ ) (Fig. 1). Figure 1D stratifies the EOR subgroups according to the intraoperative protocol.

A significant association was found between postoperative tumor volume on T2-weighted MRI images and $\Delta \mathrm{T} 2 \mathrm{~T} 1$ value $(\mathrm{rho}=0.788, p<0.001)$.

OS, PFS and MPFS. Data on 3-,5-, and 10 years estimated OS, PFS and MPFS are summarized in Table 3. Specifically, results of multivariate analysis showed that OS is independently associated with preoperative tumor growing pattern expressed by $\Delta \mathrm{T} 2 \mathrm{~T} 1(p=0.004)$, molecular class $(p=0.028)$, EOR $(p=0.018)$ (Table 4 , Fig. 2). The intraoperative protocol was not significantly associated with OS after multivariate analysis probably as a consequence of the more overwhelming effect of EOR.

Figure 2 shows the OS Kaplan-Meier curves stratified according to preoperative tumor volume, $\Delta \mathrm{T} 2 \mathrm{~T} 1, \mathrm{EOR}$, volume of residual tumor, molecular class and intraoperative protocol.

Data on 3-, 5-, and 10 years estimated OS, PFS and MPFS were also stratified according to the EOR achieved and molecular class (Tables 5, 6).

\section{Discussion}

In this retrospective study, which included 288 adult patients surgically treated for DLGG, the use of different intraoperative protocols implemented in a large single center series over time was assessed based on neurological outcomes, EOR and OS.

The most relevant results we focused on were:

(1) The evolution of the intraoperative surgical protocol over time led to an improvement in EOR.

(2) The highest percentage of total resection (EOR 100\%) was obtained amongst patients treated with Protocol 3 (DTI and fMRI data loaded in NN + AS + RTNT).

(3) Preoperative $\triangle \mathrm{T} 2 \mathrm{~T} 1 \mathrm{MRI}$ index, EOR and the molecular class are confirmed as independent predictors for OS.

(4) Tumor recurrence was also seen in patients that underwent radical resection and amongst all molecular classes.

The role of EOR. In the last decades, DLGG management paradigms have evolved from "wait and see" strategy to a more active interventional approach that aims at reducing the risk of malignant transformation. Numerous studies strongly support that a more extensive resection of DLGGs is associated with improved overall survival time and tumor progression free survival time $e^{1,3,6,11,12,24-38}$. 


\begin{tabular}{|c|c|}
\hline Parameter & Value \\
\hline No. of patients & 288 \\
\hline Age & 37.5 years $(18-74)$ \\
\hline \multicolumn{2}{|l|}{ Sex } \\
\hline Male & $168(58.33)$ \\
\hline Female & $120(41.67)$ \\
\hline \multicolumn{2}{|l|}{ Clinical onset } \\
\hline Generalized seizure & $160(55.56)$ \\
\hline Focal seizure & $80(27.78)$ \\
\hline Incidental & $36(12.5)$ \\
\hline Others (neurological deficits, mood changes) & $12(4.17)$ \\
\hline \multicolumn{2}{|l|}{ Tumor side } \\
\hline Left & $160(55.56)$ \\
\hline Right & $128(44.44)$ \\
\hline \multicolumn{2}{|l|}{ Tumor site } \\
\hline Frontal (inferior frontal gyrus, premotor cortex, middle frontal gyrus, Broca’s area) & $118(40.97)$ \\
\hline Parietal & $34(11.81)$ \\
\hline Temporal & $54(18.75)$ \\
\hline Insular & $77(26.74)$ \\
\hline Occipital & $5(1.74)$ \\
\hline Preoperative tumoral volume on $\mathrm{T} 2 \mathrm{WI}\left(\mathrm{cm}^{3}\right)$ & $40(22-68)$ \\
\hline \multicolumn{2}{|l|}{ Preoperative $\Delta \mathrm{T} 2 \mathrm{~T} 1 \mathrm{MRI}$ index, $\mathrm{cm}^{3}$} \\
\hline$\Delta \mathrm{T} 2 \mathrm{~T} 1 \mathrm{MRI}$ index & $10(0-20)$ \\
\hline$\geq 15$ & $116(40.38)$ \\
\hline$<15$ & $172(59.72)$ \\
\hline \multicolumn{2}{|l|}{ Anesthesia } \\
\hline Awake & $175(60.77)$ \\
\hline General & $113(39.23)$ \\
\hline \multicolumn{2}{|l|}{ Intraoperative surgical protocol } \\
\hline Protocol 1 & 77 (26.74) \\
\hline Protocol 2 & $137(47.57)$ \\
\hline Protocol 3 & $74(25.69)$ \\
\hline \multicolumn{2}{|l|}{ EOR \% } \\
\hline$\geq 90$ & $146(50.69)$ \\
\hline $70-89$ & $101(35.07)$ \\
\hline$<70$ & $41(14.24)$ \\
\hline Median EOR \% & $90(28-100)$ \\
\hline Protocol 1: Median EOR \% & $83(28-100)$ \\
\hline Protocol 2: Median EOR \% & $88(34-100)$ \\
\hline Protocol 3: Median EOR \% & $100(50-100)$ \\
\hline Awake surgery & $90(28-100)$ \\
\hline General anesthesia & $85(34-100)$ \\
\hline \multicolumn{2}{|l|}{ Median EOR \% in awake surgery subgroup } \\
\hline Protocol 1, only awake surgery: Median EOR \% & $85(28-100)$ \\
\hline Protocol 2, only awake surgery: Median EOR \% & $90(49-100)$ \\
\hline Protocol 3, only awake surgery: Median EOR \% & $100(50-100)$ \\
\hline \multicolumn{2}{|l|}{ Median EOR \% stratified by molecular class } \\
\hline DA IDH $1 / 2$ wt & $83(34-100)$ \\
\hline DA IDH $1 / 2 \mathrm{mt}$ & $87(28-100)$ \\
\hline OD IDH 1/2mt,1p19q cod & $92(55-100)$ \\
\hline \multicolumn{2}{|l|}{ Post-operative residual tumor volume on T2WI $\left(\mathrm{cm}^{3}\right)$} \\
\hline$<10$ & $189(65.63)$ \\
\hline $10-19$ & $51(17.71)$ \\
\hline $20-29$ & $23(7.99)$ \\
\hline$\geq 30$ & $25(8.68)$ \\
\hline Median & $5(0-125)$ \\
\hline \multicolumn{2}{|l|}{ Diagnosis WHO 2016} \\
\hline Continued & \\
\hline
\end{tabular}




\begin{tabular}{|c|c|}
\hline Parameter & Value \\
\hline DA IDH-wt & $34(11.8)$ \\
\hline DA IDH-mt & $163(56.6)$ \\
\hline OD IDH-mt 1p19q cod & $91(31.6)$ \\
\hline \multicolumn{2}{|l|}{ Clinical follow up } \\
\hline Median follow up (months) & $71(18-239)$ \\
\hline Patient deaths & $141(48.96)$ \\
\hline $\mathrm{n}^{\circ} \mathrm{w} /$ disease progression & $199(69.1)$ \\
\hline Median time to progression (months) & $46(4-194)$ \\
\hline $\mathrm{n}^{\circ} \mathrm{w} /$ malignant progression & $159(55.21)$ \\
\hline Median time to malignant progression (months) & $60(6-239)$ \\
\hline
\end{tabular}

Table 1. Clinical, radiological and molecular characteristics of the study population. Characteristics of the study population are described using means (standard deviation) or median and range for continuous variables, number of cases with relative percentages reported in parentheses for categorical variables. $\Delta T 2 T 1$ $M R I$ index volumetric difference between preoperative tumor volumes on T2 and T1 weighted MRI images, EOR extent of surgical resection, DA IDHwt Diffuse Astrocytoma Isocitrate Dehydrogenase wild type, $D A$ IDHmt Diffuse Astrocytoma Isocitrate Dehydrogenase mutated, OD IDHmt $1 p 19 q$ cod Oligodendroglioma Isocitrate Dehydrogenase mutated, $1 \mathrm{p} 19 \mathrm{q}$ codeleted.

\begin{tabular}{|c|c|c|c|c|}
\hline & General population & Protocol 1 & Protocol 2 & Protocol 3 \\
\hline Total population & 288 & 77 & 137 & 74 \\
\hline PO deficit-total & $100(34.72 \%)$ & $30(38.96 \%)$ & $47(34.31 \%)$ & $23(31.08 \%)$ \\
\hline Aphasia and hemiplegia & $3(1.04 \%)$ & $0(0 \%)$ & $1(0.73 \%)$ & $2(2.7 \%)$ \\
\hline Dysarthria & $14(4.86 \%)$ & $2(2.6 \%)$ & $6(4.38 \%)$ & $6(8.11 \%)$ \\
\hline Dysphasia & $12(4.17 \%)$ & $3(3.9 \%)$ & $5(3.65 \%)$ & $4(5.41 \%)$ \\
\hline Dysphasia and hemiplegia & $1(0.35 \%)$ & $0(0 \%)$ & $1(0.73 \%)$ & $0(0 \%)$ \\
\hline Dysphasia and hemiparesis & $1(0.35 \%)$ & $0(0 \%)$ & $0(0 \%)$ & $1(1.35 \%)$ \\
\hline Dysphasia and upper limb paresis & $2(0.69 \%)$ & $0(0 \%)$ & $0(0 \%)$ & $2(2.7 \%)$ \\
\hline Dysarthria and upper limb paresis & $4(1.39 \%)$ & $2(2.6 \%)$ & $1(0.73 \%)$ & $1(1.35 \%)$ \\
\hline Dysphasia and upper limb paresthesia & $1(0.35 \%)$ & $0(0 \%)$ & $1(0.73 \%)$ & $0(0 \%)$ \\
\hline Hemiplegia & $9(3.13 \%)$ & $4(5.19 \%)$ & $5(3.65 \%)$ & $0(0 \%)$ \\
\hline Hemiparesis & $11(3.82 \%)$ & $5(6.49 \%)$ & $4(2.92 \%)$ & $2(2.7 \%)$ \\
\hline Lower limb paresis & $11(3.82 \%)$ & $5(6.49 \%)$ & $6(4.38 \%)$ & $0(0 \%)$ \\
\hline Upper limb paresis & $12(4.17 \%)$ & $2(2.6 \%)$ & $7(5.11 \%)$ & $3(4.05 \%)$ \\
\hline Upper limb plegia & $1(0.35 \%)$ & $0(0 \%)$ & $1(0.73 \%)$ & $0(0 \%)$ \\
\hline Hypoesthesia of the Hemisoma & $1(0.35 \%)$ & $1(1.3 \%)$ & $0(0 \%)$ & $0(0 \%)$ \\
\hline Ataxia & $1(0.35 \%)$ & $1(1.3 \%)$ & $0(0 \%)$ & $0(0 \%)$ \\
\hline Lower limb paresthesia & $3(1.04 \%)$ & $0(0 \%)$ & $3(2.19 \%)$ & $0(0 \%)$ \\
\hline Lower limb paresthesia and upper limb paresis & $1(0.35 \%)$ & $0(0 \%)$ & $1(0.73 \%)$ & $0(0 \%)$ \\
\hline Parestesia of the hemisoma & $4(1.39 \%)$ & $0(0 \%)$ & $3(2.19 \%)$ & $1(1.35 \%)$ \\
\hline SMA syndrome & $8(2.78 \%)$ & $5(6.49 \%)$ & $2(1.46 \%)$ & $1(1.35 \%)$ \\
\hline 6-m deficit-total & $12(4.17 \%)$ & $5(6.49 \%)$ & $5(3.65 \%)$ & $2(2.7 \%)$ \\
\hline Aphasia and hemiplegia & $2(0.69 \%)$ & $0(0 \%)$ & $1(0.73 \%)$ & $1(1.35 \%)$ \\
\hline Dysarthria & $1(0.35 \%)$ & $1(1.3 \%)$ & $0(0 \%)$ & $0(0 \%)$ \\
\hline Dysphasia & $2(0.69 \%)$ & $0(0 \%)$ & $1(0.73 \%)$ & $1(1.35 \%)$ \\
\hline Hemiplegia & $6(2.08 \%)$ & $3(3.9 \%)$ & $3(2.19 \%)$ & $0(0 \%)$ \\
\hline Hemiparesis & $1(0.35 \%)$ & $1(1.3 \%)$ & $0(0 \%)$ & $0(0 \%)$ \\
\hline
\end{tabular}

Table 2. Neurological impairment in the immediate post-operative period and 6 months after surgery in the general population and according to intraoperative protocol. Table showing the presence of neurological impairment in the immediate post-operative period and 6 months after Diffuse Low-Grade Glioma surgery in the general population and for each intraoperative Protocol. Protocol 1: Brain mapping and Direct Electrical Stimulation. Protocol 2: Protocol 1 plus overlap of functional MRI/DTI imaging on a NeuroNavigation system. Protocol 3: Protocol 2 plus Real-Time Neuropsychological Testing. PO deficit neurological deficit in the immediate Post-Operative period, 6- $m$ deficit neurological deficit 6 months after surgery. 
A Comparison of EOR distribution by intraoperative protocol

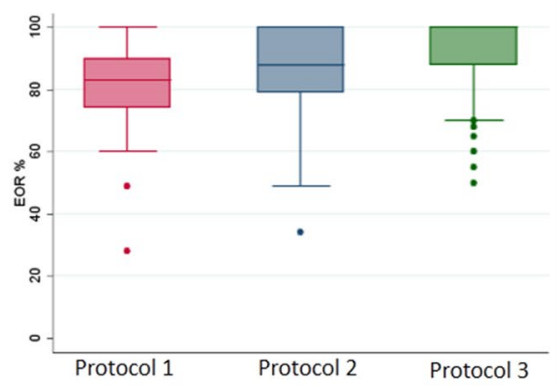

C Comparison of EOR distribution by anesthesia

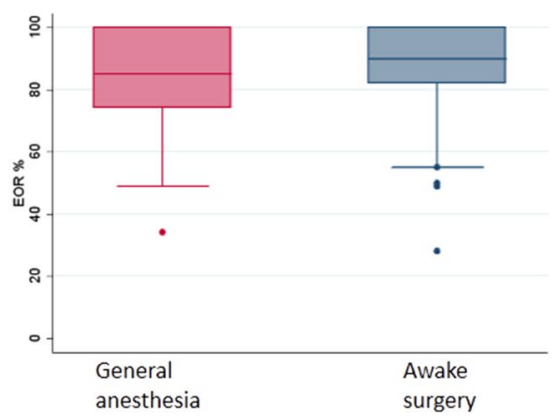

B Comparison of EOR distribution by intraoperative protocol

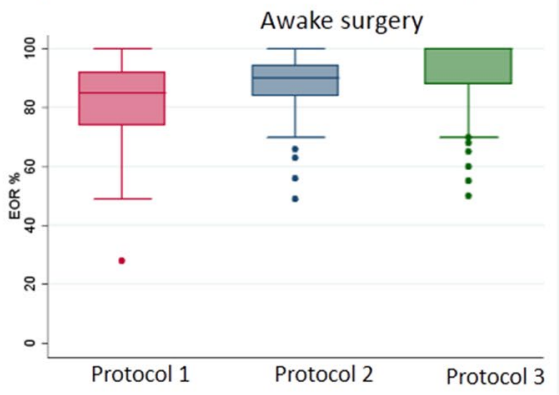

D Stratification of EOR for each intraoperative protocol

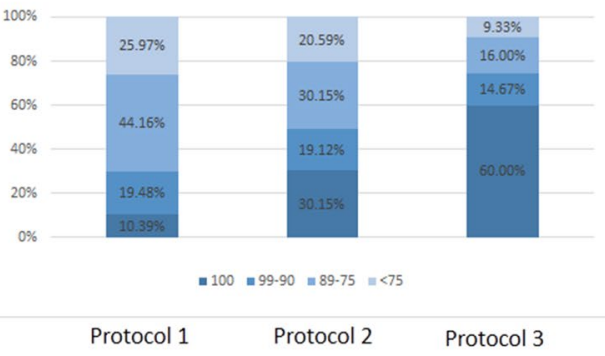

Figure 1. Difference achieved in tumor resection according to the intraoperative protocol used. (A) shows the median EOR achieved in patients operated with general anesthesia and awake craniotomy. In (B) the EOR data are stratified according to the intraoperative surgical protocol. Protocol 1 (Mapping) had a median EOR of 83\%, while Protocol 2 (Mapping + DTI + fMRI) had a median EOR of $88 \%$ and Protocol 3 had a median EOR of $100 \%$. (C) displays the median EOR achieved in the subgroup of patients who underwent awake craniotomy: Protocol 1 (Mapping) 85\%, Protocol 2 (Mapping + DTI + fMRI) 90\% and Protocol 3 100\%. The circles represent the outlier values. (D) is a bar chart representing the distribution of EOR as a categorical variable in the three surgical protocols. EOR Extent Of Resection.

\begin{tabular}{|l|l|l|l|}
\hline & $\begin{array}{l}\text { Overall survival } \\
\text { \% (95\%CI) }\end{array}$ & $\begin{array}{l}\text { Progression free survival } \\
\text { \% }(\mathbf{9 5} \% \mathbf{C I})\end{array}$ & $\begin{array}{l}\text { Malignant progression free survival } \\
\mathbf{9}(\mathbf{9 5} \mathbf{C I})\end{array}$ \\
\hline 3 years & $87.58(83.04-90.96)$ & $69.23(63.39-74.33)$ & $80.17(74.95-84.41)$ \\
\hline 5 years & $75.80(70.01-80.62)$ & $47.77(41.41-53.85)$ & $64.88(58.64-70.43)$ \\
\hline 10 years & $43.69(36.35-50.79)$ & $15.39(10.37-21.33)$ & $32.85(26.04-39.80)$ \\
\hline
\end{tabular}

Table 3. Overall survival, progression free survival and malignant progression free survival at 3,5 and 10 years after surgery. Table showing overall survival, progression free survival and malignant progression free survival at 3, 5 and 10 years after surgery.

The milestone of LGGs surgery is the concept of maximal safe resection ${ }^{5,17,39,40 .}$.

Our results confirm that EOR is the strongest independent predictor for OS in DLGG: patients who underwent radical resection ( $\mathrm{EOR}=100 \%$ ), when functionally possible, showed significant survival benefit.

Association between postoperative tumor volume on T2-weighted MRI images and preoperative $\Delta \mathrm{T} 2 \mathrm{~T} 1 \mathrm{MRI}$ index showed that the greater the preoperative $\Delta \mathrm{T} 2 \mathrm{~T} 1 \mathrm{MRI}$ index, the less extensive was the possible resection; it highlights a possible predictive correlation between $\triangle \mathrm{T} 2 \mathrm{~T} 1 \mathrm{MRI}$ index and EOR achievable as previously demonstrated ${ }^{38}$.

A major volumetric difference between T2-weighted and contrast-enhanced T1-weighted MRI sequences suggests a greater propensity of the tumor to have a diffuse growing pattern and consequently to be less resectable.

The intraoperative surgical protocol. The principal limiting factor of surgical radicality lies in the infiltrating nature of these tumors, which gradually overgrow into eloquent areas both at cortical and subcortical level ${ }^{14,40}$. A personalized anatomo-functional planning is consequently mandatory ${ }^{6,17}$.

In the modern era of glioma surgery, increasing technological advances in imaging methods (such as intraoperative NN, MRI, and ultrasonography), stimulation mapping, and/or somatosensory-evoked potential techniques, are enabling surgeons to maximize cytoreduction and minimize morbidity.

Although there is growing literature supporting the accuracy and efficiency of individual techniques and methods ${ }^{6,11,41}$, we found no studies in literature that report the analysis of multimodal integrated protocols in the same cohort of DLGGs. 


\begin{tabular}{|c|c|c|c|c|c|c|}
\hline \multirow[b]{2}{*}{ Variable } & \multicolumn{3}{|c|}{ Univariate analysis } & \multicolumn{3}{|c|}{ Multivariate analysis } \\
\hline & Odds ratio & $95 \% \mathrm{CI}$ & $p$ value & Odds ratio & $95 \% \mathrm{CI}$ & $p$ value \\
\hline Age (years) & 1.017 & $1.003-1.031$ & 0.014 & 1.010 & $0.993-1.041$ & 0.248 \\
\hline \multicolumn{7}{|l|}{ Anesthesia } \\
\hline General & 1 & & & & & \\
\hline Awake & 0.801 & $0.571-1.124$ & 0.199 & & & \\
\hline \multicolumn{7}{|l|}{ Intraoperative protocol } \\
\hline 1 & 1 & & & & & \\
\hline 2 & 0.660 & $0.465-0.937$ & 0.020 & 0.952 & $0.616-1.472$ & 0.826 \\
\hline 3 & 0.448 & $0.238-0.842$ & 0.013 & 1.174 & $0.561-2.455$ & 0.671 \\
\hline \multicolumn{7}{|l|}{ PO deficit } \\
\hline No deficit & 1 & & & & & \\
\hline Presence of neurological deficit & 1.858 & $1.322-2.610$ & $<0.001$ & 1.406 & $0.912-2.168$ & 0.123 \\
\hline \multicolumn{7}{|l|}{ 6-m deficit } \\
\hline No deficit & 1 & & & & & \\
\hline Presence of neurological deficit & 1.898 & $1.114-3.235$ & 0.018 & 0.797 & $0.397-1.600$ & 0.523 \\
\hline \multicolumn{7}{|l|}{ Clinical onset } \\
\hline Generalized seizure & 1 & & & & & \\
\hline Focal seizure & 1.395 & $0.978-1.991$ & 0.067 & 1.509 & $0.999-2.280$ & 0.051 \\
\hline Neurological impairment & 3.300 & $1.036-10.512$ & 0.043 & 1.827 & $0.397-8.422$ & 0.439 \\
\hline Incidental & 0.121 & $0.030-0.491$ & 0.003 & 0.518 & $0.117-2.288$ & 0.386 \\
\hline Headache & 1.977 & $0.621-6.293$ & 0.249 & 1.589 & $0.443-5.701$ & 0.478 \\
\hline \multicolumn{7}{|l|}{ Side } \\
\hline Left & 1 & & & 1 & & \\
\hline Right & 0.747 & $0.535-1.043$ & 0.087 & 0.944 & $0.636-1.401$ & 0.774 \\
\hline \multicolumn{7}{|l|}{ Tumor site } \\
\hline Insula & 1 & & & 1 & & \\
\hline Frontal lobe & 0.848 & $0.571-1.260$ & 0.415 & 1.087 & $0.643-1.837$ & 0.756 \\
\hline Parietal lobe & 1.267 & $0.756-2.122$ & 0.369 & 1.173 & $0.618-2.229$ & 0.625 \\
\hline Temporal lobe & 0.611 & $0.352-1.058$ & 0.079 & 0.907 & $0.476-1.728$ & 0.766 \\
\hline \multicolumn{7}{|l|}{ Histological diagnosis (WHO 2016) } \\
\hline OD IDHmt 1p19q cod & 1 & & & 1 & & \\
\hline DA IDHwt & 2.830 & $1.616-4.959$ & $<0.001$ & 2.663 & $1.271-5.583$ & 0.009 \\
\hline DA IDHmt & 1.700 & $1.149-2.516$ & 0.008 & 1.680 & $1.059-2.666$ & 0.028 \\
\hline \multicolumn{7}{|l|}{ Radiological features } \\
\hline $\begin{array}{l}\text { Preoperative tumoral volume computed on T2-weighted } \\
\text { images, } \mathrm{cm}^{3}\end{array}$ & 1.008 & $1.005-1.011$ & $<0.001$ & 0.998 & $0.991-1.005$ & 0.615 \\
\hline Preoperative MRI Index $\Delta \mathrm{VT} 2-\mathrm{T} 1$ & 1.039 & $1.030-1.048$ & $<0.001$ & 1.026 & $1.008-1.044$ & 0.004 \\
\hline Residual tumor, $\mathrm{cm}^{3}$ & 1.017 & $1.012-1.023$ & $<0.001$ & 0.996 & $0.978-1.015$ & 0.662 \\
\hline EOR (continuous variable) & 0.956 & $0.946-0.965$ & $<0.001$ & 0.975 & $0.956-0.996$ & 0.018 \\
\hline \multicolumn{7}{|l|}{ Biological features } \\
\hline p53 & 1.314 & $0.909-1.901$ & 0.147 & & & \\
\hline ATRX & 0.880 & $0.604-1.282$ & 0.506 & & & \\
\hline
\end{tabular}

Table 4. Predictors of overall survival univariate and multivariate analyses. Table showing the influence of different factors on the OS rates as per univariate survival analysis and multivariate analysis on the entire patient's cohort. ( $p$ value $<0.05$ at Log-rank test). Boldfacing represent statistically significant results $(p<0.05)$. $C I$ confidence interval, $p$ value level of marginal significance, $P O$ deficit post-operative deficit, 6- $m$ deficit deficit 6 months after surgery, $M R I$ magnetic resonance image, Index $\triangle V T 1 / T 2$ ratio between pre-operative tumoral volume on postcontrast T1-weighted and T2-weighted images, EOR extent of resection, $R T$ radiotherapy, CT chemotherapy, MGMT O6-alkylguanine DNA alkyltransferase, OS overall survival.

Volumetric studies have shown the prognostic significance of EOR for survival outcomes but have not compared the impact of the various intraoperative imaging modalities and surgical strategies in assisting/optimizing the maximal safe resection ${ }^{13,18}$.

This investigation assessed the efficacy of the combination of different techniques in surgery of DLGGs that are positioned in or close to eloquent areas both in terms of morbidity and EOR.

Specifically, neuroradiological techniques, such as fMRI and 3D reconstruction of white matter tracts, represent useful tools to preoperatively analyze the three-dimensional relationships between the tumor and the 

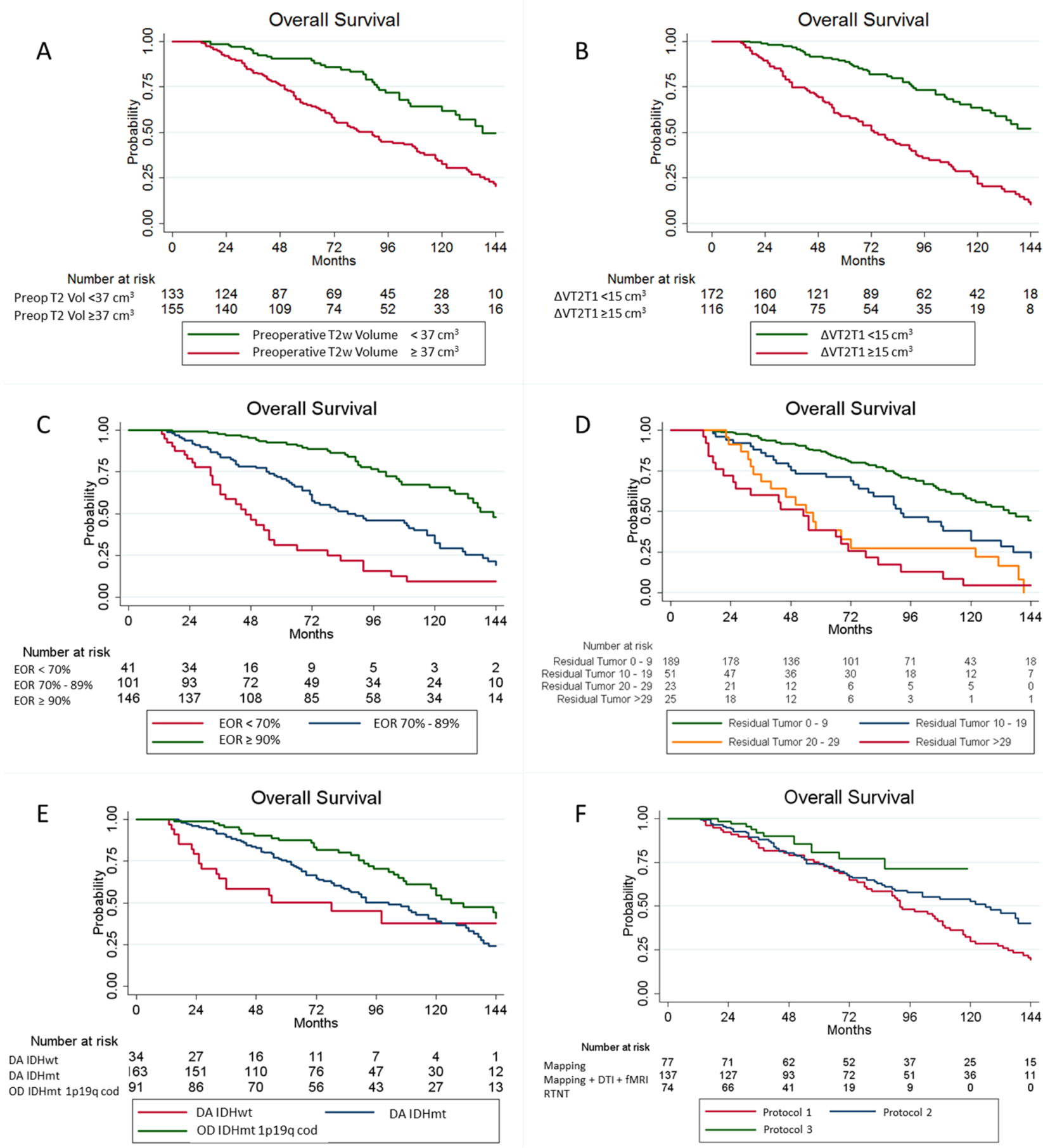

Figure 2. Kaplan-Meier curves displaying overall survival (OS) according to the preoperative tumor volume (A), preoperative tumor growing pattern, as expressed by $\Delta \mathrm{T} 2 \mathrm{~T} 1$ (B), extent of resection (C), intraoperative protocol (D), residual tumor (E) and molecular class (F). Preop T2 Vol preoperative tumor volume computed on T2-weighted images, $\Delta T 2 T 1$ preoperative tumor volume segmented on T2-weighted MRI images - preoperative tumor volume segmented on T1-weighted images, EOR extent of resectionm DA IDHwt Diffuse Astrocytoma Isocitrate Dehydrogenase wild type, DA IDHmt Diffuse Astrocytoma Isocitrate Dehydrogenase mutated, $O D$ IDHmt 1p19q cod Oligodendroglioma Isocitrate Dehydrogenase mutated, 1p 19q codeleted.

neighboring cortical functional areas and subcortical pathways, respectively ${ }^{6,18,42-44}$. Intraoperative use of a guided navigation system enriched with functional and DTI data used in Series 2 significantly increase the possible EOR. Patients in Series 1 had a mean EOR of $83 \%$, while those in Series 2 had a mean EOR of $88 \%(p<0.001)$.

Overall, the use awake mapping and RTNT (Protocol 3) resulted in a higher EOR and lower permanent deficits. The data show that Protocol 3 was superior to Protocol 1 and 2 in improving the EOR. The median EOR and the number of radical resections is hence superior in patients belonging to Protocol 3 in comparison with those 


\begin{tabular}{|l|l|l|l|}
\hline & EOR 100\% & EOR 99-85\% & EOR $<85 \%$ \\
\hline Overall survival \\
\hline 3 years & $97.72(91.18-99.43)$ & $95.46(88.36-98.27)$ & $71.61(61.58-79.46)$ \\
\hline 5 years & $94.92(86.92-98.08)$ & $84.79(75.24-90.88)$ & $52.46(41.83-62.03)$ \\
\hline 10 years & $86.58(71.80-93.92)$ & $45.28(33.52-56.31)$ & $19.21(11.28-28.74)$ \\
\hline Progression free survival & $90.83(82.49-95.31)$ & $69.93(59.27-78.30)$ & $49.26(38.99-58.73)$ \\
\hline 3 years & $70.94(58.65-80.18)$ & $49.02(38.20-58.97)$ & $26.82(18.09-36.33)$ \\
\hline 5 years & $46.49(31.41-60.25)$ & $11.28(5.47-19.44)$ & $3.76(0.77-10.95)$ \\
\hline 10 years & Malignant progression free survival & \\
\hline 3 years & $94.26(86.75-97.57)$ & $87.77(79.00-93.04)$ & $60.57(50.18-69.45)$ \\
\hline 5 years & $91.12(82.08-95.71)$ & $67.93(57.01-76.64)$ & $40.92(30.79-50.77)$ \\
\hline 10 years & $72.74(54.86-84.47)$ & $26.87(17.31-37.36)$ & $14.69(7.76-23.71)$ \\
\hline
\end{tabular}

Table 5. Overall survival, progression free survival and malignant progression free survival at 3,5 and 10 years after surgery according to extent of resection. Table showing overall survival, progression free survival and malignant progression free survival at 3, 5 and 10 years after surgery according to extent of resection. Results are displayed as percentage $(95 \%$ confidence interval). $\mathrm{EOR}=$ extent of resection.

\begin{tabular}{|c|c|c|c|}
\hline & \multicolumn{3}{|c|}{$\begin{array}{l}\text { Among patients with EOR }=100 \% \\
\%(95 \% \mathrm{CI})\end{array}$} \\
\hline & DA IDHwt & DA IDHmt & OA IDHmt 1p19q cod \\
\hline Number of patients & 12 & 45 & 37 \\
\hline \multicolumn{4}{|c|}{ Overall survival \%(95\%CI) } \\
\hline 3 years & $91.67(53.90-98.78)$ & $97.56(83.92-99.65)$ & $100.00(-)$ \\
\hline 5 years & $91.67(53.90-98.78)$ & $91.76(76.39-97.29)$ & $100.00(-)$ \\
\hline 10 years & $91.67(53.90-98.78)$ & $87.59(69.47-95.29)$ & $86.54(55.83-96.48)$ \\
\hline \multicolumn{4}{|c|}{ Progression free survival \%(95\%CI) } \\
\hline 3 years & $91.67(53.90-98.78)$ & $88.15(73.83-94.89)$ & $93.94(77.69-98.47)$ \\
\hline 5 years & $82.50(46.09-95.33)$ & $62.40(43.68-76.45)$ & $77.18(55.45-89.25)$ \\
\hline 10 years & $68.75(29.07-89.26)$ & $51.51(30.79-68.82)$ & $26.23(7.52-50.06)$ \\
\hline \multicolumn{4}{|c|}{ Malignant progression free survival \%(95\%CI) } \\
\hline 3 years & $91.67(53.90-98.78)$ & $92.85(79.43-97.64)$ & $96.67(78.61-99.52)$ \\
\hline 5 years & $91.67(53.90-98.78)$ & 85.97 (68.99-94.03) & 96.67 (78.61-99.52) \\
\hline 10 years & $91.67(53.90-98.78)$ & $71.05(42.29-87.30)$ & $70.86(41.90-87.25)$ \\
\hline
\end{tabular}

Table 6. Overall survival, progression free survival and malignant progression free survival at 3,5 and 10 years after surgery in patients with total resection, stratified according to molecular diagnosis (WHO 2016). Table showing overall survival, progression free survival and malignant progression free survival at 3, 5 and 10 years after surgery according to molecular diagnosis (WHO 2016), in patients with Extent of Resection $=100 \%$. EOR extent of resection, DA IDHwt Diffuse Astrocytoma Isocitrate Dehydrogenase wild type, DA IDHmt Diffuse Astrocytoma Isocitrate Dehydrogenase mutated, OD IDHmt 1p19q cod Oligodendroglioma Isocitrate Dehydrogenase mutated, $1 \mathrm{p} 19 \mathrm{q}$ codeleted.

belonging to Protocol 1 and $2(p<0.001)$ (Fig. 1). Indeed, RTNT used in Protocol 3 proved to be an important step of the brain mapping technique.

Throughout surgical resection, the patient performs multiple complex tasks involving the cognitive function(s) related to the brain area where the surgeon is working at that moment, thus guiding the surgical team. When a significant decrement of performance is noted, the surgeon can stop resection or move to another area, otherwise the surgical resection can be carried out without the uncertainty of "classical" negative mapping. As a matter of fact, RTNT can be defined as a continuous neuropsychological monitoring.

Thus, a multimodal integrated protocol with those techniques (brain mapping and multimodal intraoperative imaging) and strategies (AS and RTNT) can improve the chances of an extensive resection, which has repercussions on survival and recurrence. In light of that, Protocol 3 could represent a feasible support to the surgeon to safely maximize EOR in DLGGs surgery.

DES, both at cortical and subcortical levels, according to some experts of this field of surgery, represents the standard of care for DLGGs resection. It is used both for brain mapping and for monitoring neurologic performance, often in awake setting ${ }^{13}$. 
Our study showed that patients who underwent AS had a better EOR in comparison to those operated under GA $(p=0.006)$ supporting the importance of AS for DLGGs in eloquent areas. This is the sole technique enabling a direct identification of neural networks and thus maximizing the resection while minimizing the risk of permanent deficits ${ }^{39}$. RTNT is based on a continuous neuropsychological testing administered during the surgical resection in order to fill the functional information gap between two consecutive DES, thus aiming at exploiting at its maximum the potentiality of $\mathrm{AS}^{17}$.

With regards to the neurological outcomes, our results showed that patients belonging to Protocol 3 resulted in fewer late neurological deficits than those that underwent Protocol 1, even if the difference was not significant, which was mainly due to the limited sample size of this rare disease.

De Witt Hamer et al. hypothesized that AS enables more extended resection and improved tumor control, resulting in the preservation of neurological functions that can be mapped at the cost of early transient neurological deficits ${ }^{13}$. In this investigation, in line with previous studies, the simultaneous decrease in transient and permanent deficit testifies the preservation of functional subcortical pathways, ensuring the postoperative plasticity mechanism for the neurological recovery ${ }^{45,46}$.

The molecular landscape. The 2016 WHO classification incorporated molecular findings into brain tumor diagnosis. Phenotypic and genotypic parameters in the diagnosis of DLGGs (mutations in the IDH1 and IDH2 genes mutations and $1 \mathrm{p} / 19 \mathrm{q}$ co-deletion) have become of crucial importance ${ }^{47}$.

This investigation confirms the role of molecular class as an independent predictor of $\mathrm{OS}^{35,48}$. It also confirms the association between the molecular class and EOR achieved ${ }^{22}$.

The median EOR was larger in Oligodendroglioma than in Diffuse Astrocytoma IDH1/2 wild-type (92\% vs. $83 \% ; p=0.002$ ) and tended to also be larger in Diffuse Astrocytoma IDH1/2 mutated than in Diffuse Astrocytoma IDH $1 / 2$ wild-type $(87 \%$ vs. $83 \% ; p=0.05)$. This difference could be related to a different tumor growing pattern.

Unfortunately, our data showed that DLGGs recurred in some of the patients even in cases of radical resection (Table 6). Interestingly, 3 and 5 years estimated OS in patients with an EOR of $100 \%$ was higher in patients belonging to molecular class of Diffuse Astrocytoma IDH1/2 mutated and Oligodendroglioma. Otherwise, considering the 10 years estimated OS, the percentage was similar amongst Diffuse Astrocytoma IDH1/2 mutated and Oligodendroglioma, and greater for Diffuse Astrocytoma IDH1/2 wild-type.

Future studies are thus required to better investigate the rich set of pertinent genomic and molecular markers, in addition to the radiological features in the era of radiogenomics, to better understand the biological behavior of DLGGs.

Limitations. The retrospective nature of the investigation and the different treatments performed at tumor progression are the principal limitations of the present study. Nevertheless, we attempted to homogenize our data set by studying a large sample of patients based on strict inclusion criteria.

Furthermore, considering the low incidence of DLGGs, well-designed retrospective studies, even if limiting in nature, can contribute to the existing body of knowledge.

T2w-FLAIR sequences were not available for both pre- and postoperative measures for some patients. We used the late postoperative scan to minimize overestimation of postoperative tumor volume due to edema or ischemia and to minimize potential differences between sequences ${ }^{49}$, 50. Gliomas were classified according to the currently used WHO 2016 classification in this study, however, in light of the wide heterogeneity of DLGG, future investigations based on texture features from multi-parametric MRI and next generation sequences (NGS) analysis, may better clarify tumor recurrence even for those cases with a radical resection ${ }^{47,51}$.

In addition, the cornerstone of LGGs surgery is based on the concept of maximal safe resection ${ }^{5,17,39,40 .}$ Although intraoperative mapping of brain areas was shown to promote greater extent of resection and reduce functional deficits, this was shown only recently for some non-invasive techniques. We recognize that in this clinical setting the preoperative functional mapping by navigated transcraninical magnetic stimulation (nTMS) is an increasing valuable tool to stratify the individual risk of surgery-related deficits ${ }^{52,53}$. Our presurgical workup, at the moment, does not include this technique that has become an important tool in the modern surgical glioma management.

In closing, considering the higher interindividual variability of eloquent area arrangement and the wide potential displacement of functional sites, both at cortical level and subcortical pathways induced by the DLGG growing, a personal tailored multimodal integrated assessment of functional area should be developed to adopt in DLGG surgical field.

\section{Conclusions}

Technological tools are clinically useful to improve the extent of resection. Multimodal integrated approaches combining awake craniotomy, RTNT, intraoperative brain mapping and functional imaging analysis can enhance maximal safe and efficient tumor resection with possible subsequent OS benefits.

Combining volumetric studies and Next Generation Sequence investigations will provide a better understanding of DLGG evolution, paving the way for molecular neurosurgery.

Received: 8 December 2020; Accepted: 1 April 2021

Published online: 11 May 2021

\section{References}

1. Capelle, L. et al. Spontaneous and therapeutic prognostic factors in adult hemispheric World Health Organization Grade II gliomas: a series of 1097 cases: clinical article. J. Neurosurg. 118, 1157-1168. https://doi.org/10.3171/2013.1.JNS121 (2013). 
2. Clark, V. E. \& Cahill, D. P. Extent of resection versus molecular classification: What matters when?. Neurosurg. Clin. N. Am. 30, 95-101. https://doi.org/10.1016/j.nec.2018.08.006 (2019).

3. Claus, E. B. et al. Survival rates in patients with low-grade glioma after intraoperative magnetic resonance image guidance. Cancer 103, 1227-1233. https://doi.org/10.1002/cncr.20867 (2005).

4. Hervey-Jumper, S. L. \& Berger, M. S. Maximizing safe resection of low- and high-grade glioma. J. Neurooncol. 130, 269-282. https:// doi.org/10.1007/s11060-016-2110-4 (2016).

5. Ius, T. et al. An NF- $\mathrm{BB}$ signature predicts low-grade glioma prognosis: A precision medicine approach based on patient-derived stem cells. Neuro Oncol. 20, 776-787. https://doi.org/10.1093/neuonc/nox234 (2017).

6. Ius, T. et al. Low-grade glioma surgery in eloquent areas: volumetric analysis of extent of resection and its impact on overall survival. A single-institution experience in 190 patients. J. Neurosurg. 117, 1039-1052. https://doi.org/10.3171/2012.8.jns12393 (2012).

7. Lang, F. F. \& Gilbert, M. R. Diffusely infiltrative low-grade gliomas in adults. J. Clin. Oncol. 24, 1236-1245. https://doi.org/10.1200/ JCO.2005.05.2399 (2006).

8. Nitta, M. et al. Proposed therapeutic strategy for adult low-grade glioma based on aggressive tumor resection. Neurosurg. Focus 38, E7 (2015).

9. Sanai, N. How to build a neurosurgical oncology practice specializing in gliomas. Neurosurg. Clin. N. Am. 30, 129-136. https:// doi.org/10.1016/j.nec.2018.08.013 (2019)

10. Sanai, N., Chang, S. \& Berger, M. S. Low-grade gliomas in adults. J. Neurosurg. 115, 948-965 (2011).

11. Skrap, M. et al. Surgery of insular nonenhancing gliomas: Volumetric analysis of tumoral resection, clinical outcome, and survival in a consecutive series of 66 cases. Neurosurgery 70, 1081-1094. https://doi.org/10.1227/NEU.1080b1013e31823f31825be31825 (2012).

12. Smith, J. S. et al. Role of extent of resection in the long-term outcome of low-grade hemispheric gliomas. J. Clin. Oncol. 26, 1338-1345. https://doi.org/10.1200/JCO.2007.1313.9337 (2008).

13. De Witt Hamer, P. C., Robles, S. G., Zwinderman, A. H., Duffau, H. \& Berger, M. S. Impact of intraoperative stimulation brain mapping on glioma surgery outcome: A meta-analysis. J. Clin. Oncol. 30, 2559-2565. https://doi.org/10.1200/jco.2011.38.4818 (2012).

14. Mandonnet, E. et al. Silent diffuse low-grade glioma: Toward screening and preventive treatment?. Cancer 120, 1758-1762. https:// doi.org/10.1002/cncr.28610 (2014).

15. Duffau, H. Is non-awake surgery for supratentorial adult low-grade glioma treatment still feasible?. Neurosurg. Rev. 41, 133-139 (2018).

16. Duffau, H. Awake mapping is not an additional surgical technique but an alternative philosophy in the management of low-grade glioma patients. Neurosurg. Rev. 41, 689-691. https://doi.org/10.1007/s10143-10017-10937-10146 (2018).

17. Skrap, M., Marin, D., Ius, T., Fabbro, F. \& Tomasino, B. Brain mapping: a novel intraoperative neuropsychological approach. J. Neurosurg. 125, 877-887. https://doi.org/10.3171/2015.10.jns15740 (2016).

18. Mazzucchi, E., La Rocca, G., Ius, T., Sabatino, G. \& Della Pepa, G. M. Multimodality imaging techniques to assist surgery in lowgrade gliomas. World Neurosurg. 133, 423-425. https://doi.org/10.1016/j.wneu.2019.10.120 (2020).

19. Berger, M. S. \& Ojemann, G. A. Intraoperative brain mapping techniques in neuro-oncology. Stereotact. Funct. Neurosurg. 58, 153-161. https://doi.org/10.1159/000098989 (1992).

20. Ius, T., Angelini, E., Thiebaut de Schotten, M., Mandonnet, E. \& Duffau, H. Evidence for potentials and limitations of brain plasticity using an atlas of functional resectability of WHO grade II gliomas: Towards a "minimal common brain". Neuroimage 56, 992-1000. https://doi.org/10.1016/j.neuroimage.2011.03.022 (2011).

21. Louis, D. N. et al. The 2016 World Health Organization classification of tumors of the central nervous system: A summary. Acta Neuropathol. 131, 803-820. https://doi.org/10.1007/s00401-016-1545-1 (2016).

22. Cesselli, D. et al. Application of an artificial intelligence algorithm to prognostically stratify grade II gliomas. Cancers 12, 50. https:// doi.org/10.3390/cancers12010050 (2019).

23. Steyerberg, E. Clinical Prediction Models: A Practical Approach to Development, Validation, and Updating (Springer, 2009).

24. Sanai, N., Polley, M.-Y. \& Berger, M. S. Insular glioma resection: Assessment of patient morbidity, survival, and tumor progression. J. Neurosurg. 112, 1-9 (2010).

25. Majchrzak, K. et al. The assessment of prognostic factors in surgical treatment of low-grade gliomas: A prospective study. Clin. Neurol. Neurosurg. 114, 1135-1144. https://doi.org/10.1016/j.clineuro.2012.02.054 (2012).

26. Nitta, M. et al. Updated therapeutic strategy for adult low-grade glioma stratified by resection and tumor subtype. Neurol. Med. Chir. (Tokyo) 53, 447-454. https://doi.org/10.2176/nmc.53.447 (2013).

27. Snyder, L. A. et al. The impact of extent of resection on malignant transformation of pure oligodendrogliomas. J. Neurosurg. 120, 309-314. https://doi.org/10.3171/2013.10.JNS13368 (2014).

28. Coburger, J. et al. Low-grade glioma surgery in intraoperative magnetic resonance imaging: Results of a multicenter retrospective assessment of the german study group for intraoperative magnetic resonance imaging. Neurosurgery 78, 775-786. https://doi.org/ 10.1227/NEU.0000000000001081 (2016).

29. Jungk, C. et al. Prognostic value of the extent of resection in supratentorial WHO grade II astrocytomas stratified for IDH1 mutation status: a single-center volumetric analysis. J. Neurooncol. 129, 319-328. https://doi.org/10.1007/s11060-016-2177-y (2016).

30. Roelz, R. et al. Residual tumor volume as best outcome predictor in low grade glioma-A nine-years near-randomized survey of surgery vs. biopsy. Sci. Rep. 6, 32286. https://doi.org/10.1038/srep32286 (2016).

31. Eseonu, C. I. et al. Comparative volumetric analysis of the extent of resection of molecularly and histologically distinct low grade gliomas and its role on survival. J. Neurooncol. 134, 65-74. https://doi.org/10.1007/s11060-017-2486-9 (2017).

32. Eseonu, C. I., ReFaey, K., Garcia, O., Raghuraman, G. \& Quinones-Hinojosa, A. Volumetric analysis of extent of resection, survival, and surgical outcomes for insular gliomas. World Neurosurg. 103, 265-274. https://doi.org/10.1016/j.wneu.2017.04.002 (2017).

33. Hameed, N. U. F. et al. Transcortical insular glioma resection: clinical outcome and predictors. J. Neurosurg. 131, 706-716. https:// doi.org/10.3171/2018.4.JNS18424 (2018).

34. Patel, T. et al. The role of extent of resection in IDH1 wild-type or mutant low-grade gliomas. Neurosurgery 82, 808-814. https:// doi.org/10.1093/neuros/nyx265 (2018).

35. Wijnenga, M. M. J. et al. The impact of surgery in molecularly defined low-grade glioma: An integrated clinical, radiological, and molecular analysis. Neuro Oncol. 20, 103-112 (2018).

36. Bo, H. K. et al. Intraoperative 3D ultrasound-guided resection of diffuse low-grade gliomas: Radiological and clinical results. J. Neurosurg. 132, 518-529. https://doi.org/10.3171/2018.10.JNS181290 (2019).

37. Morshed, R. A. et al. Molecular features and clinical outcomes in surgically treated low-grade diffuse gliomas in patients over the age of 60. J. Neurooncol. 141, 383-391. https://doi.org/10.1007/s11060-018-03044-4 (2019).

38. Ius, T. et al. Incidental low-grade gliomas: Single-institution management based on clinical, surgical, and molecular data. Neurosurgery https://doi.org/10.1093/neuros/nyz114 (2019).

39. Duffau, H. Diffuse Low-Grade Gliomas in Adults 2nd edn. (Springer, 2017).

40. Mandonnet, E. \& Duffau, H. Understanding entangled cerebral networks: A prerequisite for restoring brain function with braincomputer interfaces. Front. Syst. Neurosci. 8, 82. https://doi.org/10.3389/fnsys.2014.00082 (2014).

41. Bello, L. et al. Intraoperative use of diffusion tensor imaging fiber tractography and subcortical mapping for resection of gliomas: Technical considerations. Neurosurg. Focus 28, E6. https://doi.org/10.3171/2009.12.FOCUS09240 (2010). 
42. Raffa, G. et al. The impact of diffusion tensor imaging fiber tracking of the corticospinal tract based on navigated transcranial magnetic stimulation on surgery of motor-eloquent brain lesions. Neurosurgery 83, 768-782. https://doi.org/10.1093/neuros/ nyx554 (2018)

43. Campanella, M., Ius, T., Skrap, M. \& Fadiga, L. Alterations in fiber pathways reveal brain tumor typology: A diffusion tractography study. PeerJ 2, e497. https://doi.org/10.7717/peerj.497 (2014).

44. de Bertoldi, F. et al. Improving the reliability of single-subject fMRI by weighting intra-run variability. Neuroimage 114, $287-293$. https://doi.org/10.1016/j.neuroimage.2015.03.076 (2015).

45. Duffau, H. \& Taillandier, L. New concepts in the management of diffuse low-grade glioma: Proposal of a multistage and individualized therapeutic approach. Neuro Oncol. 17, 332-342. https://doi.org/10.1093/neuonc/nou153 (2015).

46. Rossi, M. et al. Is supratotal resection achievable in low-grade gliomas? Feasibility, putative factors, safety, and functional outcome. J. Neurosurg. 132, 1692-1705 (2019).

47. Louis, D. N. et al. cIMPACT-NOW: A practical summary of diagnostic points from Round 1 updates. Brain Pathol 29, 469-472. https://doi.org/10.1111/bpa.12732 (2019)

48. Brat, D. J. et al. cIMPACT-NOW update 3: Recommended diagnostic criteria for "Diffuse astrocytic glioma, IDH-wildtype, with molecular features of glioblastoma, WHO grade IV". Acta Neuropathol. 136, 805-810. https://doi.org/10.1007/s00401-018-1913-0 (2018).

49. Ius, T. et al. Surgery for insular low-grade glioma: Predictors of postoperative seizure outcome. J. Neurosurg. 120, 12-23 (2014).

50. Pala, A. et al. The value of intraoperative and early postoperative magnetic resonance imaging in low-grade glioma surgery: A retrospective study. World Neurosurg. 93, 191-197. https://doi.org/10.1016/j.wneu.2016.04.120 (2016).

51. Yang, Y. et al. Optimizing texture retrieving model for multimodal MR image-based support vector machine for classifying glioma. J. Magn. Reson. Imaging (JMRI) 49, 1263-1274. https://doi.org/10.1002/jmri.26524 (2019).

52. Raffa, G. et al. The role of navigated transcranial magnetic stimulation for surgery of motor-eloquent brain tumors: A systematic review and meta-analysis. Clin. Neurol. Neurosurg. 180, 7-17. https://doi.org/10.1016/j.clineuro.2019.03.003 (2019).

53. Ille, S. \& Krieg, S. M. Functional mapping for glioma surgery, part 1: Preoperative mapping tools. Neurosurg. Clin. N. Am. 32, 65-74. https://doi.org/10.1016/j.nec.2020.08.004 (2021).

\section{Acknowledgements}

We would like to thank Mark Zeppieri MD, PhD for the scientific and English revision of the manuscript.

\section{Author contributions}

Conceptualization, T.I.; Data curation, T.I., G.P., B.T., E.M.; Formal analysis, T.I. and C.B.; Methodology, T.I. and E.M.; Supervision, T.I. and M.S.; Validation, all authors; Writing-review and editing, T.I., EM., B.T.,G.P., G.S., GM. DP., G.LR., C.B., A.O. and M.S. All authors have read and agreed to the published version of the manuscript.

\section{Funding}

This research received no external funding.

\section{Competing interests}

The authors declare no competing interests.

\section{Additional information}

Correspondence and requests for materials should be addressed to T.I.

Reprints and permissions information is available at www.nature.com/reprints.

Publisher's note Springer Nature remains neutral with regard to jurisdictional claims in published maps and institutional affiliations.

Open Access This article is licensed under a Creative Commons Attribution 4.0 International License, which permits use, sharing, adaptation, distribution and reproduction in any medium or format, as long as you give appropriate credit to the original author(s) and the source, provide a link to the Creative Commons licence, and indicate if changes were made. The images or other third party material in this article are included in the article's Creative Commons licence, unless indicated otherwise in a credit line to the material. If material is not included in the article's Creative Commons licence and your intended use is not permitted by statutory regulation or exceeds the permitted use, you will need to obtain permission directly from the copyright holder. To view a copy of this licence, visit http://creativecommons.org/licenses/by/4.0/.

(C) The Author(s) 2021 